\title{
Módulos Parlantes para Impulsar los Hábitos de Higiene Bucal y Lavado de Manos
}

\section{Talking Modules to Promote Oral Hygiene and Hand Washing Habits}

\author{
Adiela Ruiz Gómez y María Alejandra Gonzalez Bernal
}

Recibido: 30/enero/2021

Aceptado: 6/abril/2021

Publicado: 16/abril/2021

\&yis País

${ }^{12}$ Colombia

\section{IIII. Institución \\ ${ }^{12}$ Universidad Cooperativa de Colombia}

\section{$\lambda_{\Omega}$ Correo Eletrónico}

1ªdiela.ruizg@campusucc.edu.co ${ }^{2}$ maria.gonzalezbe@campusucc.ed u.co

\section{(D) ORCID}

${ }^{1}$ https://orcid.org/0000-0003-2011-2555 ${ }^{2}$ https://orcid.org/0000-0002-6299-2135

\section{Citar así: : APA / IEEE}

Ruiz-Gómez, A. \& Gonzalez-Bernal, M (2021). Módulos parlantes para impulsar los hábitos de higiene bucal y lavado de manos. Revista Tecnológica-Educativa Docentes 2.0, 11(1), 140-145. https://doi.org/10.37843/rted.v11i1.203

A. Ruiz Gómez y M. Gonzalez Bernal, "Módulos parlantes para impulsar los hábitos de higiene bucal y lavado de manos", RTED, vol. 11, n. ${ }^{\circ} 1$, pp. 140-145, abr. 2021.

\section{Resumen}

La caries dental presenta una alta prevalencia, se previene con medidas de higiene bucal; los profesores presentan prácticas sobre cepillado dental e higiene de manos que definirán en los alumnos de corta edad comportamientos base de su salud en el futuro. El objetivo fue diseñar dos módulos parlantes como apoyo a las practicas sobre hábitos de higiene bucal y lavado de manos en profesores de un centro intercultural en Bogotá, 2020. Metodología. Se hizo una investigación cualitativa, en una muestra no probabilística, homogénea, de ocho profesores responsables de enseñar a niños provenientes de etnias indígenas urbanas. Se utilizó como técnica una entrevista no estructurada, posteriormente se hizo análisis de contenido de las frases transcritas textualmente en las respuestas, como base para proponer una herramienta didáctica de apoyo mediada por las TIC con la finalidad de incentivar hábitos saludables, considerando el contexto intercultural para predecir los resultados a mediano y largo plazo. Resultados. Se encontró en los profesores la definición de boca y manos desde el punto de vista anatómico, su uso para lo cotidiano además de servir en el desarrollo personal; los profesores propusieron el diseño de dos módulos parlantes en dialecto de las etnias de los niños del centro intercultural. Conclusiones. la comprensión sobre salud bucal y lavado de manos de los profesores permitió proponer conjuntamente el diseño de dos módulos parlantes para impulsar los hábitos de higiene bucal y lavado de manos.

Palabras clave: Salud bucal,, cepillado, etnias, TIC.

Dental caries has a high prevalence, it is prevented with oral hygiene measures; The teachers present practices on tooth brushing and hand hygiene that will define the basic behaviors of their health in the young students in the future. The objective was to design two talking modules to support practices on oral hygiene and hand washing habits in teachers of an intercultural center in Bogotá, 2020. Methodology. A qualitative research was carried out in a homogeneous, non-probabilistic sample of eight teachers responsible for teaching children from urban indigenous ethnic groups. An unstructured interview was used as a technique; later on, the content analysis of the phrases transcribed verbatim in the answers was made, as a basis to propose a support didactic tool mediated by ICT in order to encourage healthy habits, considering the intercultural context to predict medium and long-term results. Results. The definition of mouth and hands was found in the teachers from the anatomical point of view, their use for everyday life as well as serving in personal development; the teachers proposed the design of two speaking modules in the dialect of the ethnic groups of the children from the intercultural center. Conclusions. Teachers' understanding of oral health and handwashing made it possible to jointly propose the design of two speaking modules to promote oral hygiene and handwashing habits.

Keywords: Transition, impact, distance learning, technological tools, bilingualism. 


\section{Introducción}

En Colombia el Sistema General de Seguridad Social en Salud (SGSSS), tiene como finalidad promover, restaurar y mantener la salud de la población sin excepción alguna (Ministerio de Salud y Protección Social Colombia, 2014, p.11), por lo cual el Ministerio de Salud incluye en este plan a la población indígena, quienes han sido excluidos en diferentes campos ya sea por su cultura, tradición, actitudes y costumbres (Olivar Rojas, 2020,p.148); buscando así garantizar el cumplimiento de sus derechos, y la igualdad ante los sistemas de salud.

En Bogotá, se ha identificado el mayor número de población indígena proveniente de diferentes ciudades del país y otras naciones vecinas, en su mayoría pertenecientes a los pueblos Wayuu, Nasa y Pastos (DANE, 2019, p.19) (MinSalud, 2020, p.4). En el caso de la población infantil se busca un enfoque de derechos con una perspectiva diferencial y de inclusión social, a partir de crear espacios de recuperación y saber ancestral, implicándose los hábitos que estos presentan (Alcaldía Mayor de Bogotá, 2011, p.9). Un hábito hace referencia a actos y prácticas tomados por costumbre (Sánchez-Ojeda \& De Luna-Bertos, 2015, p.1911), para un bienestar físico, mental y social, interviniendo en la vida cotidiana del ser humano; los hábitos en general se pueden tomar desde diferentes perspectivas, incluyéndose hábitos saludables de higiene bucal, lavado de manos, seguridad y redes de apoyo (GarcíaUbaque, 2011, p.19).

La continua llegada de familias de diferentes etnias a Bogotá, por procesos de movilización interna debido a múltiples factores, ha obligado la búsqueda de estrategias para la aplicación de la política pública de primera infancia, a partir del enfoque diferencial étnico; una de estas estrategias corresponde a la implementación de jardines infantiles para el acceso de los niños a un entorno donde se incorpore el enfoque diferencial étnico, priorizando en su nutrición, cuidado $\mathrm{y}$ formación. (Bogotá humana, 2012, p.23).

Las casas de pensamiento intercultural surgen ante la necesidad de contar con espacios para el acompañamiento de los niños y por iniciativa de algunas autoridades tradicionales, con el fin de impulsar el pensamiento intercultural ante el debilitamiento de la identidad propia; se encaminan a la formación en saberes ancestrales y en la apropiación de la historia de sus pueblos y cultura. (Delgadillo, García y Sandoval, 2013, p.161).

El centro de pensamiento intercultural Payacua, se ubica en el barrio Ricaurte, localidad los Mártires, integra a los pueblos indígenas de la ciudad, víctimas de discriminación $\mathrm{y}$ segregación racial; encontrando dos de los grupos con mayor población indígena como lo son Nasa y Pastos (Casa de pensamiento intercultural Payacua, 2011,p.2); en ellos, se han detectado problemas en hábitos saludables como higiene bucal y lavado de manos, a pesar de contar con un análisis de la situación en salud y de estar incluidas estas etnias en los diferentes proyectos y estrategias enfocadas a mejorar la salud oral, propuestas por el gobierno de Bogotá (Ministerio de Salud, 1999,p.65).

Para facilitar la comunicación y colaboración en el diseño de entornos de aprendizaje, se han utilizado métodos en donde se da voz e involucra a grupos de personas, relacionadas con esta actividad, entre ellos, colectivos vulnerables (Gros \& Maniega, 2018,p.3).Uno de estos métodos es el diseño participativo donde se desarrolla empatía entre los involucrados (Gros \& Durall, 2020 p.13), pudiéndose generar elementos tecnológicos que se materializan desde la manera como se percibe una situación; esta forma de trabajo involucra un proceso de dialogo de saberes que permite entender preferencias, deseos y necesidades en escenarios contextualizados (Acosta \& Garcés, 2016,p.20).

En el ámbito de educación para la salud, la participación comunitaria, es referida como un medio para abordar los problemas de salud desde la toma de decisiones conjuntas, (Palmar-Santos, 2014) por tanto el diseño participativo, favorece formas de expresión en la que se busca incluir diversas actitudes, experiencias y saberes. Por otra parte, en el diseño de tecnología educativa, el involucramiento de las personas permite la democratización en el diseño de elementos tecnológicos. (Feenberg, 2017, p.51). con los que se busca mejorar la comunicación. 
Por medio de un estudio cualitativo y entrevistas semiestructuradas a profesores del centro intercultural, se exploraron y describieron prácticas sobre hábitos de higiene bucal y de manos, con el fin de analizar esta información y proponer desde la mirada de los profesores el diseño de módulos parlantes mediados por las TIC (Jiménez, 2019, p.1) para impulsar los hábitos de higiene bucal y lavado de manos. El objetivo de la presente investigación fue diseñar dos módulos parlantes como apoyo a las practicas sobre hábitos de higiene bucal y lavado de manos en profesores de un centro intercultural en Bogotá durante el año 2020.

\section{Metodología}

Para el desarrollo de esta investigación se utilizó un enfoque cualitativo, teniendo en cuenta aspectos de participación acción; El estudio se realizó con ocho (8) profesores en el centro intercultural Payacua en el período noviembre de 2019 - agosto 2020. El procedimiento se realizó según las etapas de una investigación cualitativa: preparatoria, trabajo de campo, analítica e informativa tomando algunos elementos de la participación -acción. Se basó en la información dada por los profesores del centro intercultural, con el fin de comprender el contexto educativo con relación a la salud bucal e higiene de manos, siendo objeto de aprendizaje y apto para la investigación cualitativa el incluir a los profesores, debido a la influencia que tienen en los hábitos saludables de los niños pertenecientes a la institución.

Se incluyeron, según participación voluntaria en el estudio con firma del consentimiento informado. Se les comunicó a los integrantes las razones para ser seleccionados en este proceso investigativo. Además, se les dio la posibilidad del acercamiento personal entre participantes e investigadores, mediante una actividad que permitió eliminar barreras para lograr una participación activa. Fue aplicada una encuesta no estructurada acerca del significado del uso de las manos y boca en la cotidianidad, para ello se tuvo una guía con los temas a cubrir, los términos a usar y el orden de las preguntas que a continuación se detallan: ¿qué es la boca?, ¿para qué se usa?, ¿cuál es la importancia de la boca?, ¿qué son las manos?, ¿para qué se usan?, ¿cuál es la importancia de las manos? Las preguntas se seleccionaron según su relevancia en el contexto y una previa revisión bibliográfica.

Una de las investigadoras recolecto la información de lo manifestado por los profesores, para luego codificar las respuestas que fueron plasmadas en una matriz de vaciado de la información, posteriormente se llevó a cabo el análisis de contenido de las respuestas. Sobre la base de lo expresado por los profesores/cuidadores se llegó conjuntamente al diseño de dos módulos parlantes: uno para el lavado de manos y el otro para el cepillado dental.

\section{Resultados}

Se aplicó la entrevista semiestructurada a ocho (8) profesores, de los cuales el 87,5\% siete (7) correspondió a mujeres y $12,5 \%$ (1) a hombres. Hubo un hombre y cinco mujeres mestizos y dos mujeres pertenecientes al grupo étnico Pasto, con edad promedio de 36 años (D.E 10 años). Cuando se les preguntó: ¿qué es la boca? y ¿cuál es su importancia?, definieron básicamente la boca desde el punto de vista anatómico y en cuanto a su uso se relacionó con la expresión, la fisiológica y la higiene, con respuestas como: E1: "Es la parte de nuestro cuerpo humano, que hace parte del sistema digestivo, permite articular fonemas y palabras para poder comunicarnos a través del habla". Y la importancia estuvo relacionada con el órgano perceptivo y la deglución, con respuestas como: E2: "Importante porque de ella detecta los alimentos ingeridos estén aptos para nuestro cuerpo, con ayuda del visto, la lengua, los dientes" también con la relación interpersonal. E1: "Nos permite comunicarnos y expresarnos".

Las manos fueron definidas desde el punto de vista anatómico y referente a su uso en expresión, información y herramienta dando como resultado: E1: "Es una parte fundamental de nuestro cuerpo, que está compuesta por los dedos y las uñas, sirven para sostener los objetos, expresarnos". Su importancia se centró en acciones relacionadas con actividades cotidianas, como medio de defensa y de expresión para ligar las ideas con sensaciones y acciones, como resultado: E1: "Realizamos la mayoría de nuestras funciones y acciones con las 
manos" E3: "La importancia de esas manos es defendernos ante alguna agresión" E4: "Para lo es la más importante en la expresión corporal" Basado en las respuestas encontradas se realizó una nueva reunión para diseñar la pieza comunicativa conjunta con los profesores.

Se diseñaron dos módulos, cada figura se realizó en el programa CorelDraw Graphics Suite 2020, de acuerdo con el esquema entregado por las investigadoras y diseñados por los profesores del centro intercultural; cada uno de ellos mide de $20 \mathrm{~cm}$ de ancho x $20 \mathrm{~cm}$ de largo x $10 \mathrm{~cm}$ de fondo. En la parte frontal presenta las frases "siempre limpias para enfermedades evitar", "Mis dientes deben brillar" y "Casa de Pensamiento Intercultural"; el primero de ellos, contiene una imagen correspondiente a una mano abierta de color rosado animada sobrepuesta en una gota de agua de color azul y con fondo blanco (Figura1).

\section{Figura 1}

Diseño para el módulo parlante "Lávate las manos",

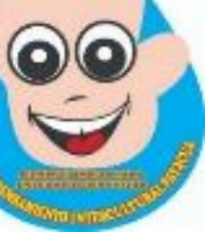

Nota. Siempre limpias para enfermedades evitar, elaborado por profesores e investigadoras (2020).

El segundo módulo consta de la imagen de un perrito animado con un cepillo de dientes (Fig. 2 ), que insta mediante un sensor de sonido a realizar el cepillado en dialecto de las etnias a la que pertenecen los niños del centro intercultural.

\section{Figura 2}

Diseño para el módulo parlante "Es hora de cepillarte"

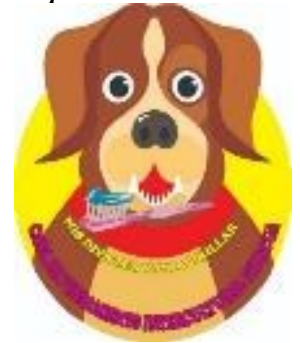

Nota. Mis dientes van a brillar, elaborado por profesores e investigadoras (2020).

\section{Discusión}

El objetivo de una estrategia educativa en salud es trabajar sobre las conductas, como lo sostiene (Palmar Santos,2014, p.81) de tal forma que se desarrollen en las personas y comunidades comportamientos positivos para configurar actitudes y estilos de vida saludables; La modificación de los comportamientos de salud requiere un enfoque pedagógico que capacite a las personas para darse cuenta de las conductas inadecuadas.

Tomando lo dicho por (Rodríguez, Páez, Altamirano et al,2017,p.3), bajo la premisa en el ámbito escolar, el profesor es el principal gestor de salud ante el escolar al comunicar y facilitar el aprendizaje de hábitos saludables, como el lavado de manos y el cepillado dental, se realizó la presente investigación; Se partió del análisis de estas prácticas en los profesores del centro Intercultural Payacua, se utilizó la entrevista semiestructurada como técnica para la identificación y valoración de los problemas y necesidades de quienes van a estar inmersos en una estrategia educativa para la salud, como lo afirma la Organización mundial de la Salud ( OMS/OPS, 1990, p.25).

Al analizar la utilidad de las manos, se destaca en los profesores respuestas acordes con lo aprendido en su formación pedagógica. Se aprecia que, si bien las identifican como parte del cuerpo y la utilidad como herramienta de aprehensión y comunicación, existen deficiencias en identificarlas como una de las principales vías en la transmisión de gérmenes. (OMS,2017, p 5) y en aspectos relacionados con el concepto del lavado de las manos como prevención en la transmisión de microorganismos. Otro aspecto importante, es el de considerar las manos para demostrar afecto, situación que puede favorecer, como lo afirman (Molina, Perez,2016, p.217) procesos interpersonales al interior del centro educativo para crear condiciones favorables en el aprendizaje de hábitos saludables.

Como bien se dijo anteriormente, en las etapas tempranas escolares, es importante la colaboración de los profesores en la detección de malos hábitos en los que puedan estar incurriendo los niños; este momento, es oportuno para la formación de hábitos básicos autocuidado 
y protección de la salud bucal como lo afirma (Díaz \& Hidalgo, 2011, p.2). Sin embargo, los estudios sobre información concerniente a los hábitos de higiene oral en profesores de niños de preescolar son relativamente escasos. Pese a que el contexto profesional y social de los profesores esta dado en un jardín intercultural, todos los participantes de la investigación consideran la boca como una de las partes más importantes del cuerpo humano, al reconocer cómo a través de ella se pueden ingerir los alimentos, hablar, reír, cantar y comunicarse, dejando de lado la importancia de asearla.

Se consideró diseñar dos módulos parlantes, como base de un prototipo para apoyar el hábito del cepillado en los niños, de tal manera para lograr una comprensión más profunda de esta temática, para que ellos puedan ejercer activamente su papel de promotores de salud oral en este espacio educativo.

\section{Conclusiones}

El uso de métodos participativos en el diseño de piezas comunicativas mediadas por las TIC, permiten un mayor conocimiento y comprensión de las prácticas en salud, desde las características de los grupos étnicos, situación que facilita proponer artefactos tecnológicos para mejorar hábitos saludables como cepillado dental y lavado de manos. El profesional de la salud debe saber comunicar y facilitar procesos de aprendizaje en las comunidades, desde una visión participativa, respetando el contexto y percepción de la realidad de los receptores de su acción pedagógica, centrándose en los conocimientos y creencias que conforman las actitudes.

El diseño y desarrollo de los artefactos tecnológicos para mejorar hábitos saludables, requiere del profesional en salud habilidades en el manejo individual y grupal desde la comunicación como su principal herramienta. Se diseñó una pieza comunicativa como estrategia educativa en salud para el apoyo de los hábitos saludables de cepillado de dientes y lavado de manos, desde el significado de los profesores.

Se requiere desarrollar el prototipo y evaluarlo como herramienta didáctica para estimular el niño o niña, a través de actividades donde se vean inmersos los sentidos para reforzar el aprendizaje y promover el seguimiento de instrucciones sobre estos hábitos saludables, facilitándole al pequeño, el refuerzo de conocimientos adquiridos a través de sus profesores.

\section{Reconocimiento}

A las estudiantes Tatiana Flórez Duarte y Michelle Alejandra Restrepo Díaz quienes, desde el semillero de salud pública, aceptaron con gran motivación formarse como investigadoras bajo una metodología innovadora que les otorgó poner en práctica todo lo aprendido.

\section{Referencias}

Acosta V, G. L., \& Garcés M, Á. (2016). El diálogo de saberes en comunicación: reconfiguraciones de la formación y de la investigación. Anagramas -Rumbos y sentidos de la comunicación-, 15(29), 17-23. http://www.scielo.org.co/scielo.php?script=sci_arttext\& pid=S1692-25222016000200001\&lng=en\&tlng=es.

Alcaldía Mayor de Bogotá D.C. (2011). Política de infancia y adolescencia en Bogotá D.C. 2011-2021. https://www.integracionsocial.gov.co/index.php/politica s-publicas/lidera-sdis/politica-publica-de-infancia-yadolescencia\#: : text=Vigencia\%3A\%202011\%20\%E2 $\% 80 \% 93 \% 202021 . \&$ text $=$ Objetivo $\% 20$ General $\% 20$ de $\%$ 201a\%20Pol\%C3\%ADtica,en\%20ejercicio\%20de \%20su s\%20derechos.

Alcaldía Mayor de Bogotá D.C. (2011). Plan de desarrollo 2012$2016 . \quad$ Bogotá humana. http://www.sdp.gov.co/transparencia/informacioninteres/otras-publicaciones/plan-de-desarrollo-20122016

Fonseca, A. (2016). Casa de Pensamiento Payacua. Blog Educación Integral, Arte y Ciudadanía. http://ciberciudadanias.blogspot.com/2016/04/casa-depensamiento-payacua.html

Departamento Administrativo Nacional de Estadística (DANE) (2019). Población Indígena de Colombia. Resultados del censo nacional de población y vivienda 2018. https://www.dane.gov.co/files/investigaciones/boletines/ grupos-etnicos/presentacion-grupos-etnicos-2019.pdf

Guido, S., García, D., Lara, G., Jutinico, M., Benavides, A., Delgadillo, I., Sandoval, B., \& Bonilla, H. (2013). Educación Inicial y diversidad cultural. Una experiencia de formación de maestros de los jardines infantiles indígenas de Bogotá. En: Experiencias de Educación Indígena en Colombia: entre prácticas pedagógicas y politicas para la educación de grupos étnicos.Universidad Pedagógica Nacional.

Díaz, Y., \& Hidalgo, I. (2011). Intervención educativa sobre salud bucal en maestros de enseñanza primaria. Revista Médica Electrónica. 33(1), 52-59. 
Feenberg, A. (2017). A Critical Theory of Technology. En: The Handbook of Science and Technology Studies (pp. 635663). Cambridge.

Garcia-Ubaque, J. C. (2011). Hábitos saludables de los trabajadores de una institución hospitalaria pública de alta complejidad en Bogotá. [tesis de maestría, Universidad Nacional de Colombia]. Repositorio UN. https://repositorio.unal.edu.co/handle/unal/7670

Gros, B., \& Maniega, D. (2018). Co-Designing Apps to Support the Learning Needs of Migrants in Barcelona (Spain). Journal of Interactive Learning Research,29(3), 465-480. https://bit.ly/2Jae5LY

Gros, B., \& Durall, E. (2020). Retos y oportunidades del diseño participativo en tecnología educativa. Edutec. Revista Electrónica De Tecnología Educativa, (74), 12-24. https://doi.org/10.21556/edutec.2020.74.1761

Jiménez Pitre, I. A. (2019). Las tendencias digitales en la educación. Revista Tecnológica-Educativa Docentes 2.0,7(2),122.

https://ojs.docentes20.com/index.php/revistadocentes20/article/view/18

Molina, N., \& Pérez N. (2006). El clima de relaciones interpersonales en el aula un caso de estudio. Paradigma 27(2),193-219.

http://ve.scielo.org/scielo.php?pid=S1011225120060002 00010\&script $=$ sci_abstract

Olivar Rojas, A.F. (2020). Políticas públicas y enfoques diferenciales: aproximaciones desde la interculturalidad y la democracia radical Iztapalapa Revista de Ciencias Sociales y Humanidades. 41(88),139-162. http://dx.doi.org/10.28928/ri/882020/aot1/olivarrojasa

Organización Mundial de la Salud. (2012). Higiene de las manos: ¿por qué, ¿cómo, ¿cuándo? Programa de Control de Infecciones. https://www.who.int/gpsc/5may/tools/ES_PSP_GPSC1 Higiene-de-las-Manos_Brochure_June-2012.pdf

Organización Mundial de la Salud/Organización Panamericana de la Salud (1990). Evaluación para el planeamiento de programas de educación para la salud. Guía para técnicos medios y auxiliares. Organización Mundial de la Salud y Organización Panamericana de la Salud. http://iris.paho.org/xmlui/bitstream/handle/123456789/3 283/Evaluación para el planeamiento de programas de educación para la salud.pdf?sequence $=1$

Rodríguez, F., Páez, R., Altamirano, E., Paraguay, F., Rodríguez, J., \& Calero, S. (2017). Nuevas perspectivas educativas orientadas a la promoción de la salud. Revista Cubana de Educación Médica Superior. 32(4),1-11. http://scielo.sld.cu/scielo.php?script=sci_arttext\&pid=S0 864-21412017000400025\&lng=pt\&nrm $=$ iso

Ministerio de Salud (1999). III Estudio Nacional de Salud Bucal. https://www.visitaodontologica.com/ARCHIVOS/ARC HIVOS-

NORMAS/Salud\%20Publica_P_y_P/II_ESTUDIO_NA CIONAL_SALUD_BUCAL.pdf

Ministerio de Salud y Protección Social Colombia. (2014). Aseguramiento al Sistema General de Seguridad Social en Salud.
igital/RIDE/VP/DOA/RL/cartillas-de-aseguramiento-alsistema-general-de-seguridad-social-en-salud.pdf

MinSalud. (2020). Boletines poblacionales: Población indígena oficina de promoción social. Bogotá, Colombia. https://www.minsalud.gov.co/sites/rid/Lists/BibliotecaD igital/RIDE/DE/PS/boletines-poblacionales-poblacionindigena.pdf

Palmar-Santos A.M. (2014). Métodos educativos en salud. Elsevier.

Sánchez-Ojeda, M. A., \& De Luna-Bertos, E. (2015). Hábitos de vida saludable en la población universitaria. Nutrición Hospitalaria, $31(5)$, 1910-1919. http://dx.doi.org/10.3305/nh.2015.31.5.8608

https://www.minsalud.gov.co/sites/rid/Lists/BibliotecaD 